\title{
The variations in light and color curves and possible short period oscillations of the binary AU Serpentis ${ }^{\star}$
}

\author{
Zong-yun $\mathbf{L i}^{1}$, Yue-rong Ding ${ }^{1}$, Zhou-sheng Zhang ${ }^{2}$, and Yu-lan $\mathbf{L i}^{2}$ \\ 1 Department of Astronomy, Nanjing University, Nanjing 210093, China \\ 2 Yunnan Observatory, Kunming 650011, China
}

Received August 11, 1997; accepted January 26, 1998

\begin{abstract}
We have photometrically monitored AU Ser for five years. It is considered to be a binary in poor thermal contact (Kaluzny 1986) or in detachment (Djurasevic 1993). The addition of new minimum times slightly modified the light ephemeris of Binnendijk (1972). The value and sign of the O'Connell effect have been changing with time. A remarkable variation of the difference between minimum depths in light curves has taken place in the period from 1991 to 1995 . These variations in light curves are not expected by TRO theory but reminiscent of the situation in VW Cep. Our data, along with Binnendijk's, show a variation in color curves especially for 1995 observations. The variations in light and color curves might be straightforwardly explained by the distortion of starspot(s). Possible oscillations with short periods and shortly coherent times have been detected, which is unusual for such a binary.
\end{abstract}

Key words: binaries: close — stars: activity — stars: oscillations — stars: individual: AU Ser

\section{Introduction}

The binary AU Ser was observed visually and photographically by Soloviev (1951) and Huth (1964) respectively. The first photoelectric observations were performed by Binnendijk (1972). Hrivnak (1993) obtained its radial velocity curve using the cross-correlation technique. Kaluzny (1986) analyzed Binnendijk's data. The pronounced asymmetry in the light curves was attributed to the effect of a hot spot located at the neck between the components, and the points in the phase interval between 0.08 and 0.46 were rejected from his analysis. AU Ser was identified as a poor thermal contact binary predicted by

Send offprint requests to: Zong-yun Li, e-mail: zyli@nju.edu.cn

* All tables are only available in electronic form at the CDS via anonymous ftp to cdsarc.u-strasbg.fr (130.79.128.5) or via http://cdsweb.u-strasbg.fr/Abstract.html the thermal relaxation oscillation (TRO) theory of Lucy \& Wilson (1979). For the same observational data, on the other hand, Djurasevic (1993) assumed that the asymmetry is due to RS CVn-type activity and he obtained a detached photometric solution. Obviously, these assumptions leading to very different solutions must be more sufficiently justified. For this reason more observations are necessary before modeling.

AU Ser has been photoelectrically monitored for five years. In this paper we report the results of these observations. A description of the observations is given in the next section; In Sects. 3 and 4 we show the variations in period, light curves and color curves, respectively. The finding of short period oscillations is described in Sect. 5. Finally we summarize the results.

\section{Observations}

We observed AU Ser photoelectrically, using the 1-meter reflector equipped with an EMI 6256 photomultiplier of the Yunnan Observatory, China, in three sets of two nights in April 1991, April 1992 and May 1995, respectively. The two filters employed are the following: a corning 5030 of stock thickness cemented with $2 \mathrm{~mm}$ Schott GG-13 for the blue region, and a corning 3389 of stock thickness for the yellow region.

We adopted the same comparison and check stars as those used by Binnendijk except for the 1991 observations in which they were shown in the finder chart in $\mathrm{Li}$ et al. (1992). The procedures of extinction corrections and of transferring into the $U B V$ system were made. The probable error of a single measurement was estimated to be $0.015 \mathrm{mag}$ for the 1991 observations, 0.012 and $0.016 \mathrm{mag}$ for $V$ and $B$ band in 1992, and 0.016 and $0.017 \mathrm{mag}$ for the two bands in 1995. The Heliocentric Julian Days at the start and at the end of the observation runs and the numbers of observations for each color are given in Table 1. The observations in 1992 and 1995 are listed in Table 2a and $2 \mathrm{~b}$ for yellow and blue light respectively, while those in 


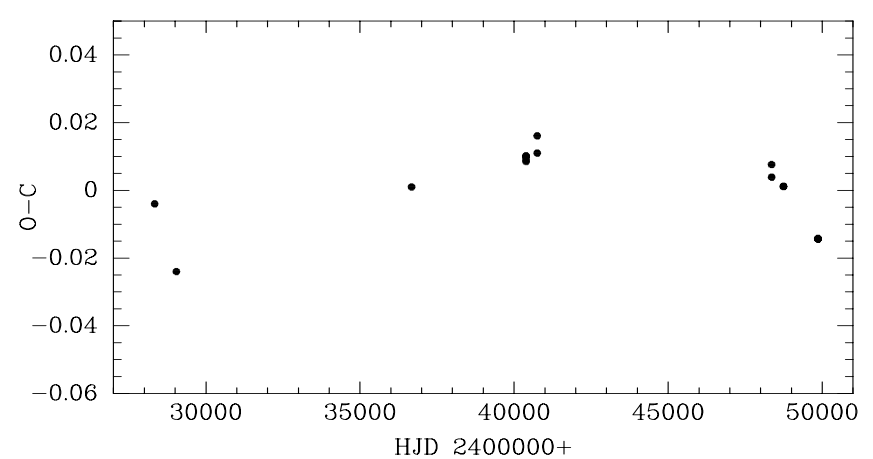

Fig. 1. O-C diagram of AU Ser

1991 have been published in Li et al. (1992). The normal points producing the light curves in Fig. 2 can be found in Table 3 through 5 . All tables are presented in electronic form.

\section{Variation in light curves}

During the observations four primary minimum times (HJD 2448356.3364 \pm 0.0003 , HJD 2448743.2192 \pm 0.0003 , HJD 2449860.1891 \pm 0.0003 and HJD 2449861.3487 \pm $0.0004)$ and three secondary minimum times (HJD 2448357.3015 \pm 0.0004 , HJD 2448742.2528 \pm 0.0004 , and HJD $2449861.1552 \pm 0.0004)$ have been obtained. New times of minimum light, together with those listed in Binnendijk (1972), give the linear ephemeris

MIN I = HJD2440748.8491+0.38650014 E.

Computed residuals have been shown in Fig. 1. O-C diagram suggests a possible variation of the orbital period. However, we are prevented from investigating the variation in detail by the absence of observations from 1938 to 1959 and from 1970 to 1991.

The data were phased according to the updated ephemeris and binned in phase intervals of 0.02 . The light curves of AU Ser are shown in Fig. 2, together with Binnindijk's observations. Although previous data are obtained with distinct instrumental systems, they are still helpful to show the relative variations between heights of two maxima or depths of two minima. Relative to 1969 curves, the others have been displaced by appropriate distances along the ordinate (tick marks are separated by $0.1 \mathrm{mag}$ ) for clarity. From Fig. 2 the considerable variations in the light curves can be seen:

\subsection{The variation of $O^{\prime}$ Connell's effect}

According to Binnendijk (1972), the maximum (MAX I) following primary minimum (MIN I) is always higher than the other (MAX II) in 1969 and 1970, i.e. O'Connell effect is positive although the author found rather rapid variations in the shape of its light curves. In our light curves obtained in 1991, the effect becomes negative. MAX I is 0.039 and 0.046 mag lower than MAX II for $V$ and
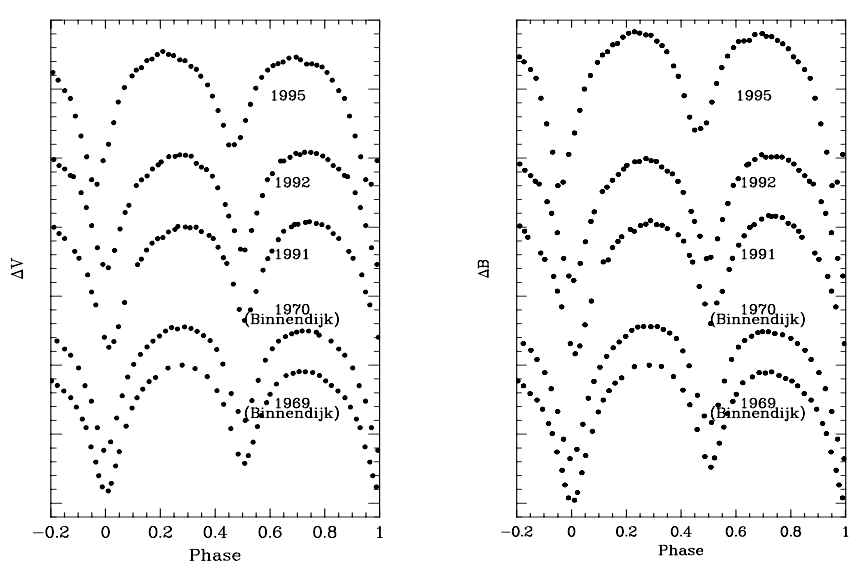

Fig. 2. The light curves of AU Ser. Relative to the light curves of 1969 (Binnendijk), the others have been displaced by the appropriate distances along the ordinate (tick marks are separated by $0.1 \mathrm{mag})$, for clarity

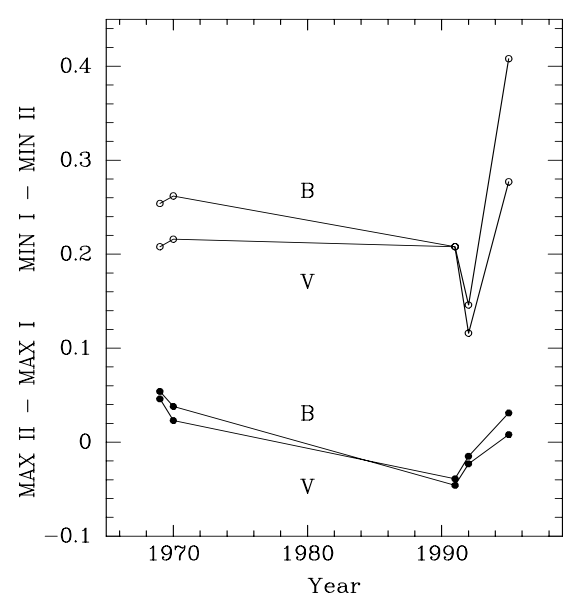

Fig. 3. The variations of heights of maxima (filled circles) and depths of minima (open circles) for $V$ and $B$ bandpasses

$B$ bandpass respectively. In 1992, MAX I is still lower than MAX II although the differences are only 0.020 and $0.029 \mathrm{mag}$. After three years, however, the situation has reversed again. MAX I becomes 0.037 and 0.012 mag higher than MAX II for both bandpasses respectively. These variations (filled circles in Fig. 3) have shown a great resemblance to those of VW Cep and are not expected by the TRO theory.

\subsection{Variation of the difference between minimum depths}

In Binnendijk's light curves, the differences are about 0.208 and $0.254 \mathrm{mag}$ in $V$ and $B$ bandpass respectively in 1969, and 0.216 and $0.262 \mathrm{mag}$ in 1970 (open circles in Fig. 3). The light curves in 1991 show the differences to be 0.208 and 0.210 mag. Both values decreased to 0.116 and 0.146 mag in 1992, which is similar to those of a typical W UMa type contact system, especially for $V$ light. However, the values in 1995 increased to 0.277 and 0.408 mag for the two bandpasses, which makes the star look more like 


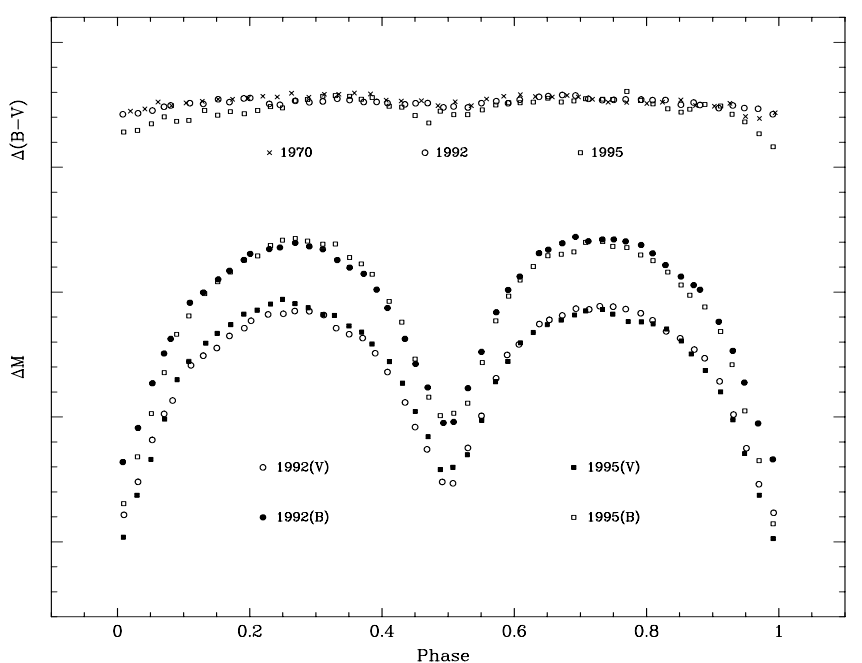

Fig. 4. The color curves in 1970, 1992 and 1995 and the light curves in 1992 and 1995 without any displacement along the ordinate. All the primary minima were moved to phase zero for comparison

an EB-system. It is most unlikely that a real change in the temperature of a component of the system has taken place in the interval of three years.

\section{Variation in color}

Since the 1991 observations were made using different comparison star, only the 1992 and 1995 observations in which the same instruments and the same comparison star were used are directly comparable. The light curves in these two years are shown in Fig. 4, where observed minima were marked with phase zero, without any displacement along the ordinate. We find that the largest variation appears around phase zero, especially for blue light.

Unlike magnitude difference, the difference in color between program and comparison stars is less affected by the instruments. The color curves of 1970, 1992 and 1995 observations are also shown in Fig. 4 (top). A common feature of them is that there are deeper or shallower dips around phases of 0 and 0.5 . The depths of the dips have been changing with time. In 1995 the depth at phase zero reaches 0.2 mag. More details can be seen in a close inspection, e.g. the color of AU Ser in 1970 is a little redder at MAX II than MAX I, the reverse situation takes place in 1992, and the star in 1995 is more or less severely "reddened" except for the phase range from 0.25 to 0.4 .

The variation in color directly reflects the variation in effective temperature. It is very unlikely that a component in this system changes much of its temperature as a whole in only three years. A possible explanation is that the appearance of a big starspot or relatively stable group of spots can reduce the star's effective temperature and produce such a effect, which is supported by the fact that light curves (Figs. 2 and 4) are not very smooth. In Fig. 5 the separation of unfolded light curves for $V$
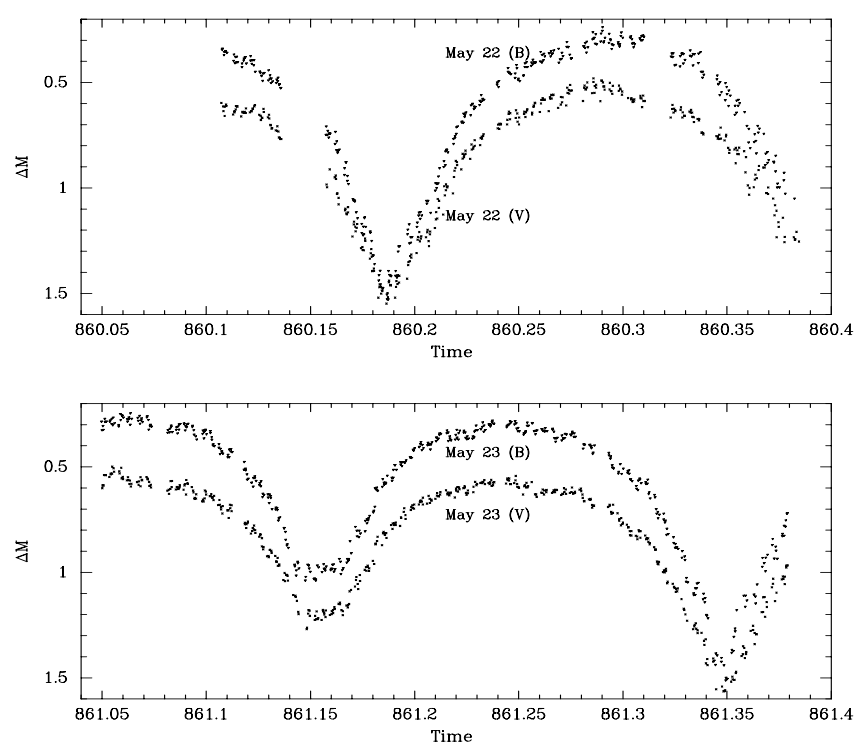

Fig. 5. The observational time series (unfolded light curves) of AU Ser in 1995, which show fluctuation, especially for the yellow on May 22 (lower curve in upper panel), with an amplitude much larger than the observational error (0.016 mag)

and $B$ bandpasses vanishes slightly before (not at!) the primary minimum on May 22, 1995 (upper panel), which can be naturally attributed to the distortion of a starspot. A qualitative analysis of intensive photometry and spectrophotometry will be able to provide a priori knowledge for modelling about the property, number and location of the starspot(s).

Although it is well-known that virtually all well observed W UMa type stars do show some changes in their light curves, the theoretical explanation is not exactly known. As far AU Ser, the heights of maxima and the depths of minima have been radically changing. We have not accumulated enough observations to study period variation, to infer its undisturbed light curves and then to derive reliable orbital elements. For this reason, no modeling was attempted in this paper and much more extended observations will be continued.

\section{Short period oscillations}

In the time series of 1995 observations, especially those in the first night (May 22), a fluctuation with an average amplitude of about 0.05 mag can be seen (Fig. 5). In order to make sure that the fluctuation is intrinsic, we subtract the variation caused by orbital motion of the binary from the time series. The binned light curves in Fig. 2 result from the orbital motion with an effect of starspots (if any). Having subtracted the interpolated values of the smooth light curves, the residual series have been shown in Fig. 6 . In the corner of each panel the letter refers to the bandpass used in the observation and the number 1 or 2 stands for the first or second night when the observation was performed. From the figure we more clearly find the fast 

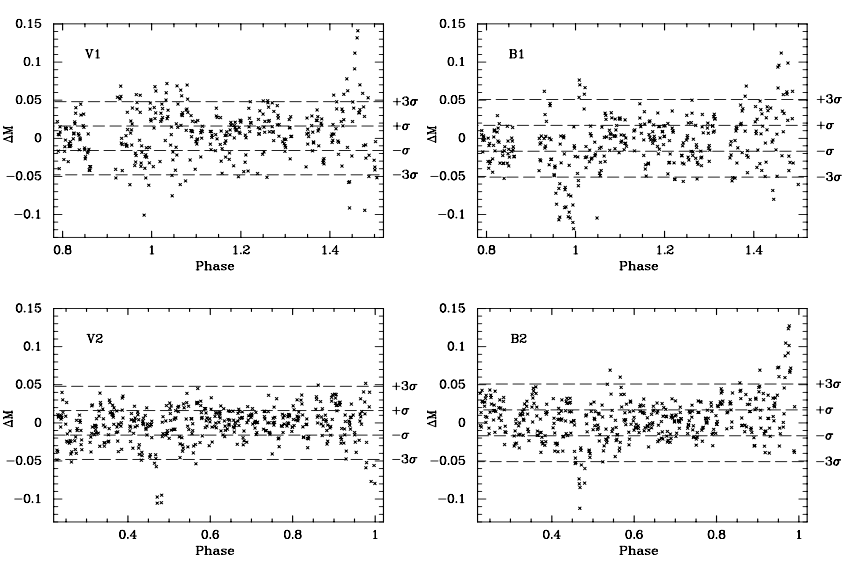

Fig. 6. The residual series in which the eclipse effect has been removed. In the upper panels (May 22 observation) we clearly find that the fluctuation has a larger amplitude, particularly around phase 1, suggesting that it should be intrinsic

variation with amplitude much larger than the probable errors of the observations, especially for $V$ bandpass of the first night observation. Moreover, it seems that the amplitude of the fluctuation is larger around the phase zero where the secondary transits the primary. If it is true, one can look for the source of the undulation from the secondary. It is most likely that the source may be the starspot(s) which caused large light and color variations and the undulation.

FFT analysis for the subtracted-data of the observation on 1995 May 22 indicates that there may exist the spikes at frequencies of 0.0003 and $0.008 \mathrm{~Hz}$. The additional spikes at $0.0049,0.0052 \mathrm{~Hz}$ in blue light and a spike around $0.005 \mathrm{~Hz}$ and a small hump around $0.0027 \mathrm{~Hz}$ in yellow light can be seen (Fig. 7). We appear to have detected the oscillations with periods of 55 and 2 minutes (coferent time is only days) and the quasi-periodic oscillations with periods around 6 and 3 minutes. The FFT spectra of May 23 observation are noisier. The spikes and the hump are drowned out, which is consistent with the lower dispersion in the lower panels of Fig. 6. However, a common feature in the FFT spectra of two night observations is the rise in power towards lower frequencies.

In order to test the reality of the peaks, we employed the CLEAN method to analyze the same data and we obtained the same results. Each panel of Fig. 7 looks like power spectra of cataclysmic variables of which some show quasi- periodic and/or coherent oscillation and the most have nonperiodic, predominantly low-frequency flickering (e.g. Robinson \& Nather 1979; Patterson 1981). To our knowledge, these types of variations have not been detected in other W UMa stars. The exciting and attenuating mechanisms of the oscillations should be investigated.

\section{Summary}

From our observations along with Binnendijk's (1972), we come to the following conclusions:
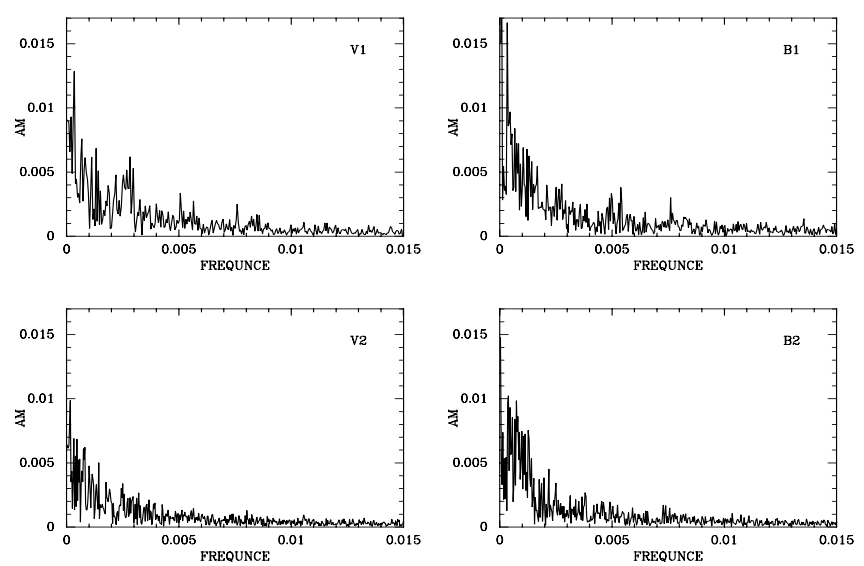

Fig. 7. The power spectra of the data shown in Fig. 6, which indicates that some short period oscillations seem to be detected on May 22, 1995 (two upper panels)

1. The light curves of AU Ser show the VW Cep or SW Lac type variation in the following aspects: i) The variation and reversion of O'Connell effect. It is positive in 1969 and 1970, negative in 1991 and 1992, and positive again in 1995. and ii) The variation of difference between minimum depths. The difference is the smallest in 1992, while the largest in 1995.

2. The color curves show variable dips around phases 0 and 0.5 . The variation in light is accompanied by the variation in color. In 1995 observation these variations are particularly significant, where remarkable dimming and severe reddening simultaneously take place around primary minimum.

3. The possible short period oscillations have been definitely detected in AU Ser on May 22 and marginally on May 23, 1995, which is unusual for such a binary.

The results reported reveal AU Ser to be a puzzling system.

Acknowledgements. We would like to thank our referee Dr. Kaluzny for his comments and helpful suggestions. Many thanks should be given to Optical Astronomy Lab., Chinese Academy of Science for scheduling the observations and providing travel grant. This work is supported by the grant 19733001 from the National Science Foundation of P.R. China.

\section{References}

Binnendijk L., 1972, AJ 77, 603

Djurasevic G., 1993, Ap\&SS 206, 207

Hrivnak B.J., 1993, in Pacific Rim Colloquium on New Frontiers in Binary Star Research, Leung K.-C. and Nha I.-S. (eds.). San Francisco: Astronomical Society of the Pacific, p. 269-274

Huth H., 1964, Mitt. Sonneberg 2, No. 5, 126

Kaluzny J., 1986, Acta Astron. 36, 113

Li Z.Y., et al., 1992, IBVS 3802

Lucy L.B., Wilson R.E., 1979, ApJ 231, 502

Patterson J., 1981, ApJS 45, 517

Robinson E.L. Nather R.R., 1979, ApJS 39, 461

Soloviev A.V., 1951, Variable Stars 8, 65 